\title{
Heavy gravitino and split SUSY in the light of BICEP2
}

\author{
JiJi Fan, Bithika Jain and Ogan Özsoy \\ Department of Physics, Syracuse University, \\ Syracuse, NY 13244, U.S.A. \\ E-mail: jijifan1982@gmail.com, bjain@syr.edu, oozsoy@syr.edu
}

ABSTRACT: High-scale supersymmetry (SUSY) with a split spectrum has become increasingly interesting given the current experimental results. A SUSY scale above the weak scale could be naturally associated with a heavy unstable gravitino, whose decays populate the dark matter $(\mathrm{DM})$ particles. In the mini-split scenario with gravitino at about the $\mathrm{PeV}$ scale and the lightest $\mathrm{TeV}$ scale neutralino being (a component of) DM, the requirement that the DM relic abundance resulting from gravitino decays does not overclose the Universe and satisfies the indirect detection constraints demand the reheating temperature to be below $10^{9}-10^{10} \mathrm{GeV}$. On the other hand, the BICEP2 result prefers a heavy inflaton with mass at around $10^{13} \mathrm{GeV}$ and a reheating temperature at or above $10^{9} \mathrm{GeV}$ with some general assumptions. The mild tension could be alleviated if SUSY scale is even higher with the gravitino mass above the $\mathrm{PeV}$ scale. Intriguingly, in no-scale supergravity, gravitinos could be very heavy at about $10^{13} \mathrm{GeV}$, the inflaton mass scale, while gauginos could still be light at the $\mathrm{TeV}$ scale.

KEYwords: Cosmology of Theories beyond the SM, Supersymmetric Standard Model

ARXIV EPRINT: 1404.1914 


\section{Contents}

1 Introduction 1

2 Gravitino and wino relic abundance $\quad 2$

2.1 Primordial gravitino relic abundance 3

2.2 Wino relic abundance from gravitino decays 4

$\begin{array}{lll}3 & \text { Indirect detection constraints } & 6\end{array}$

4 Implications of the BICEP2 result $\quad 8$

$\begin{array}{llr}4.1 \text { Basics of tensor-to-scalar ratio } & 9\end{array}$

$\begin{array}{ll}4.2 & \text { Implication for reheating temperature } \\ \end{array}$

$\begin{array}{lll}5 & \text { Implications for SUSY } & 11\end{array}$

6 Conclusions and outlook $\quad 12$

$\begin{array}{ll}\text { A Gravitino from inflaton decay } & 13\end{array}$

\section{Introduction}

Supersymmetry (SUSY) has long been a favorite theoretical framework of physics beyond the Standard Model (SM). However, given the current null results of all SUSY searches, if SUSY is realized in Nature, it is unclear at what scale it will manifest itself. At the moment, theoretical studies of SUSY fall into two broad catalogues: one direction is to still focus on weak-scale natural SUSY and design non-trivial structures of flavor and Higgs sectors to evade the direct search constraints and explain the observed Higgs mass. The other direction is take seriously high-scale fine-tuned SUSY, in particular, split SUSY, with scalars heavier than gauginos. The virtues of this approach include simplicity, automatic amelioration of SUSY flavor and CP problems, preservation of gauge coupling unification and the lightest neutralino being a dark matter (DM) candidate. The idea of split SUSY, in particular, mini-split with scalars one-loop factor heavier than gauginos, was actually predicted a while ago by the simplest version of anomaly mediation $[1,2]$ (and later by a wide variety of moduli mediation scenarios [3-8]). Since 2003, split SUSY has started to be taken as a viable possibility despite the presence of a fine-tuned EWSB and gained more attention recently given the increasing tension between data and naturalness [7-21].

In split SUSY, the high SUSY breaking scale could naturally lead to a heavy unstable gravitino. In the mini-split scenario based on anomaly mediation, there is a loop factor separating the gravitino and gaugino mass scales with gravitino at about $\left(10^{2}-10^{3}\right) \mathrm{TeV}$ and gaugino at the $\mathrm{TeV}$ scale. In this scenario, the neutralino DM particles produced 
by late-time gravitino decays could not annihilate efficiently and thus inherit the number density of the gravitinos which adds to its thermal number density. During the reheating era, the thermal scattering of the SM superpartners contributes (at least part of) the gravitino primordial relic abundance, which is approximately proportional to the reheating temperature $T_{R}$. Consequently the requirement that the neutralino DM does not overclose the Universe sets an interesting upper bound on $T_{R}$ as a function of DM mass. This upper bound could be tightened if wino is (a component of) DM. Indirect detection looking for excesses in the photon continuum spectrum or a monochromatic photon line sets a strong bound on allowed wino DM relic abundance for the whole mass range assuming NFW or Einasto DM profiles [22, 23]. The bound could be relaxed if the Milky Way DM distribution near the galactic center deviates considerably from the standard DMonly $N$-body simulation predications. However, the bound does not necessarily disappear entirely. For example, even if the Milky Way DM profile has a significant core with a radius of $1 \mathrm{kpc}$, light non-thermal wino with mass below $400 \mathrm{GeV}$ as a single-component DM is excluded [23]. We will present the derivation of the upper bound on $T_{R}$ from the constraints of the relic abundance of neutralino DM, in particular, wino DM in section 2 and section 3 .

On the other hand, the discovery of $B$-mode by the BICEP2 collaboration gives us some clues of the inflation scale [24]. The observation could be fit by a lensed $\Lambda \mathrm{CDM}$ plus tensor model with a tensor-to-scalar ratio $r=0.2_{-0.05}^{+0.07}$. Such a large $r$ prefers large field inflation with a heavy inflaton and very likely a high reheating temperature. We will present estimates of inflaton mass scale and reheating temperature in section 4.

We find that in the mini-split scenario based on anomaly mediation, $T_{R}$ is bounded to be at or below $10^{9}-10^{10} \mathrm{GeV}$ while the BICEP2 data prefers $T_{R}$ to be around or above $10^{9} \mathrm{GeV}$. The BICEP2 result has some tension with the mini-split scenario with a heavy gravitino. In other words, the BICEP2 result favors a splitting between gravitinos and gauginos larger than the loop factor predicted by anomaly mediation. Intriguingly, if SUSY breaking is tied up with gravity, e.g., through the Scherk-Schwarz mechanism, gravitinos could be as heavy as $10^{13} \mathrm{GeV}$, which is the same mass scale of the inflaton inferred from the BICEP2 result while gauginos could still be light at the TeV scale. The implications for SUSY scales will be discussed in section 5. See refs. [25-33] for some other recent discussions of implications of the BICEP2 result for SUSY.

We conclude in section 6 and present a discussion of gravitinos from inflaton decays in the appendix.

\section{Gravitino and wino relic abundance}

In this section, we first review different mechanisms generating the primordial gravitino relic abundance in the early Universe. Then we discuss the relic abundance of wino DM from gravitino decays. Notice that most of the discussions also apply to other neutralino DM scenarios such as higgsino DM. The main point we want to emphasize is that: for gravitinos at or below the $\mathrm{PeV}$ scale, the neutralino DM relic abundance has an irreducible non-thermal contribution which scales linearly with the inflaton reheating temperature 
$T_{R}$; in particular, requiring DM relic abundance not overclose the Universe restricts the reheating temperature to be below $\left(10^{10}-10^{9}\right) \mathrm{GeV}$ for DM mass in the range (100 GeV - $1 \mathrm{TeV}$ ); for gravitino much above the $\mathrm{PeV}$ scale, the neutralino DM relic abundance is almost UV insensitive, meaning that it is almost independent of $T_{R}$.

\subsection{Primordial gravitino relic abundance}

As a superpartner of the graviton, the gravitino couples to all supermultiplets with gravitational interaction strength. In an $R$-parity conserving scenario, an unstable gravitino always decays to a particle and its superpartner. Decay of a gravitino will always produce a lightest superparticle (LSP) as all the other produced superparticles will cascade down to the LSP. The decay width of an unstable gravitino is given by

$$
\Gamma_{3 / 2} \approx 2.0 \times 10^{-23} \mathrm{GeV}\left(\frac{N_{G}}{12}\right)\left(\frac{m_{3 / 2}}{100 \mathrm{TeV}}\right)^{3},
$$

where $m_{3 / 2}$ is the gravitino mass and $N_{G}$ is the number of degrees of freedom gravitino decays to. In the split SUSY scenarios with all gauginos lighter than the gravitino and the squarks heavier than the gravitino, $N_{G}=12 .{ }^{1}$

There could be several different origins of the primordial gravitino relic abundance, $\Omega_{3 / 2} h^{2}$. One comes from scattering processes of MSSM particles in the thermal bath [3436]. This contribution approximately scales linearly with the inflaton reheating temperature $T_{R}$. The higher $T_{R}$ is, the larger the gravitino relic abundance is. We will use the following approximate formula for the gravitino yield:

$$
Y_{3 / 2}^{U V} \approx \sum_{i=1}^{3} y_{i} g_{i}^{2}\left(T_{R}\right) \ln \left(\frac{k_{i}}{g_{i}\left(T_{R}\right)}\right)\left(\frac{T_{R}}{10^{9} \mathrm{GeV}}\right)
$$

where $y_{1,2,3}=(0.653,1.604,4.276) \times 10^{-13}, k_{1,2,3}=1.266,1.312,1.271$ and $g_{1,2,3}\left(T_{R}\right)$ are gauge couplings of $\mathrm{SM}$ gauge group $\mathrm{U}(1)_{\mathrm{Y}}, \mathrm{SU}(2)_{\mathrm{W}}, \mathrm{SU}(3)_{\mathrm{c}}$ evaluated at $T_{R}$ respectively [34]. The small $y$ 's originate from $T_{R} / M_{p}$ with $M_{p}$ the reduced Planck scale. Compared to the formula given in [34], we neglected a contribution at the order of $\left(M_{i}^{2} / m_{3 / 2}^{2}\right)$ with $M_{i}$ the gaugino masses. The yield given in (2.2) leads to a gravitino relic abundance

$$
\Omega_{3 / 2}^{U V} h^{2} \approx 5.1 \times 10^{-2}\left(\frac{m_{3 / 2}}{1 \mathrm{TeV}}\right)\left(\frac{T_{R}}{10^{9} \mathrm{GeV}}\right),
$$

where we evaluated temperature dependent variables in eq. (2.2) at $T_{R}=10^{9} \mathrm{GeV}$. In the numerical evaluation in section 3 , we include the full temperature dependence.

Another potential important contribution to the gravitino relic abundance comes from the decays of superpartners that are still in thermal equilibrium with the post-inflationary thermal bath [37]. ${ }^{2}$ When the temperature of the primordial plasma drops around the

\footnotetext{
${ }^{1}$ The squarks could be lighter than the gravitino in the split SUSY scenarios and then $N_{G}$ is larger. However, it will not change much our discussions and results.

${ }^{2}$ This contribution exists only when $m_{s}>m_{3 / 2}$, where $m_{s}$ denotes the SUSY scalar mass. Besides, the thermal equilibrium requires reheating temperature to be $T_{R}>m_{s}$.
} 
SUSY scalar masses, which we will take to be around the same scale, decays of the scalars to gravitinos could also generate a potentially non-negligible contribution to the gravitino relic abundance. This is the "freeze-in" mechanism [38]. When the temperature drops below the scalar masses, the number density of SUSY scalars is suppressed exponentially, $e^{-m_{s} / T}$ and freeze-in stops. The freeze-in contribution is independent of the UV physics, particularly the reheating temperature $T_{R}$ [39]. The gravitino yield from freeze-in is

$$
\begin{aligned}
Y_{3 / 2}^{F I} & \simeq \frac{405}{4 \pi^{4}} \sqrt{\frac{5}{2}} \frac{M_{p}}{g_{*}^{3 / 2}} \sum_{i} g_{i} \frac{\Gamma_{i}}{m_{i}^{2}} \\
& \approx 1.6 \times 10^{-16}\left(\frac{200}{g_{*}}\right)^{3 / 2}\left(\frac{100 \mathrm{TeV}}{m_{3 / 2}}\right)^{2} \sum_{i} g_{i}\left(\frac{m_{i}}{1000 \mathrm{TeV}}\right)^{3},
\end{aligned}
$$

where we approximated $g_{*}\left(m_{i}\right) \simeq g_{S *}\left(m_{i}\right)$ and $\Gamma_{i}=(1 / 48 \pi)\left(m_{i}^{5} /\left(m_{3 / 2}^{2} M_{p}^{2}\right)\right)$ as the partial decay width of scalar $i$ to the gravitino. Here, $g_{i}$ denotes degrees of freedom of SUSY scalar $i$ with mass $m_{i}$. The yield in eq. (2.4) leads to a gravitino relic abundance

$$
\Omega_{3 / 2}^{F I} h^{2} \approx 1.1 \times 10^{-2}\left(\frac{100 \mathrm{TeV}}{m_{3 / 2}}\right) \sum_{i} g_{i}\left(\frac{m_{i}}{1000 \mathrm{TeV}}\right)^{3} .
$$

It is clear that the gravitino relic abundance $\Omega_{3 / 2} h^{2}$ from the freeze-in contribution is highly sensitive to the scalar superpartner masses $m_{i}$ as it scales as $\sim m_{i}^{3}$.

The total gravitino abundance is just a sum of the thermal scattering (eq. (2.3)) and freeze-in (eq. (2.5)) contributions

$$
\Omega_{3 / 2} h^{2}=\Omega_{3 / 2}^{U V} h^{2}+\Omega_{3 / 2}^{F I} h^{2} .
$$

Before ending this section, we want to mention that there could be other modeldependent sources of primordial gravitino relic abundance. For example, decay of inflaton itself could also produce a sizable gravitino relic abundance. The contribution to gravitino relic abundance from inflaton decays depends on the structure of the dynamical SUSY breaking sector and could be problematic [40, 41]. However, as discussed in [42, 43], gravitino production from inflaton decay can be suppressed if there exists a hierarchy between the mass scales of the inflaton and the field whose $F$-term VEV breaks SUSY spontaneously. In the discussions below, we will not include this model dependent contribution. We refer the reader to the appendix A for more details of gravitinos from inflaton decays.

\subsection{Wino relic abundance from gravitino decays}

In this section, we will specify the neutralino DM to be wino yet the discussions hold for other neutralino DM such as higgsino DM. We will also focus on gravitino with mass above $10 \mathrm{TeV}$ so that its lifetime is shorter than a second and its decays do not spoil the successful Big Bang Nucleosynthesis [44].

The relic abundance of wino DM is a sum of the thermal contribution and the nonthermal contribution from gravitino decays. The non-thermal contribution could be computed numerically by solving the Boltzmann equations eq. (2.1) - (2.3) in ref. [45]. The 
primordial gravitino relic abundance in eq. (2.2) and (2.4) discussed in the previous section is an input to the Boltzmann equations. In solving the Boltzmann equations, we took $g_{*}(T)$ and $g_{*, s}(T)$ from a table in the DarkSUSY code [46]. ${ }^{3}$ As the Sommerfeld effect becomes important for heavy winos $[45,47,48]$, we computed the temperature-dependent value of $\left\langle\sigma_{\text {eff }} v\right\rangle$ from a preliminary version 1.1 of the DarkSE code [49], taking into account not only the Sommerfeld effect but also co-annihilation among different wino species. ${ }^{4}$ As an input to this code, we have used the two-loop splitting between the neutral and charged winos from ref. [50]. For wino masses of about a $\mathrm{TeV}$ and temperatures around a $\mathrm{GeV}$, the Sommerfeld enhancement can be as large as 3 in $\left\langle\sigma_{\text {eff }} v\right\rangle$.

The non-thermal contribution to wino relic abundance from gravitino decays changes parametrically when the gravitino mass $m_{3 / 2}$ increases. For large gravitino mass, the wino LSP produced from the gravitino decays can annihilate effectively due to the high temperature of the plasma at the time of gravitino decay. More specifically, we find that $\mathrm{DM}$ annihilation is efficient for $m_{3 / 2} \gtrsim 10^{4} \mathrm{TeV}$. This can be seen by estimating the "decay temperature" as in [45]

$$
T_{3 / 2} \equiv\left(\frac{10}{g_{*}\left(T_{3 / 2}\right) \pi^{2}} M_{\mathrm{pl}}^{2} \Gamma_{3 / 2}^{2}\right)^{1 / 4} \approx 2.2 \mathrm{GeV}\left(\frac{75.75}{g_{*}\left(T_{3 / 2}\right)}\right)^{1 / 4} \sqrt{\frac{N_{G}}{12}}\left(\frac{m_{3 / 2}}{10^{4} \mathrm{TeV}}\right)^{3 / 2}
$$

At such high temperature, winos produced from the gravitino decays annihilate rapidly, reducing the number density $n_{\tilde{W}}$ down to a critical value $n_{c, \tilde{W}} \simeq 3 H /\left.\left\langle\sigma_{\text {eff }} v\right\rangle\right|_{T=T_{3 / 2}}$ at which winos can no longer annihilate. This critical value $n_{c, \tilde{W}}$ behaves as an attractor in determining relic abundance of wino LSP, making it independent of the primordial gravitino relic abundance. In this case, the wino relic abundance is given as

$$
\begin{aligned}
\Omega_{\tilde{W}}^{(\mathrm{ann})} h^{2} & \left.\approx m_{\tilde{W}} \frac{3 H}{\left\langle\sigma_{\mathrm{eff}} v\right\rangle s}\right|_{T=T_{3 / 2}}\left(\frac{h^{2}}{\rho_{c, 0} / s_{0}}\right) \\
& \approx 0.12\left(\frac{75.75}{g_{*}\left(T_{3 / 2}\right)}\right)^{1 / 4}\left(\frac{m_{\tilde{W}}}{1 \mathrm{TeV}}\right)\left(\frac{1.2 \times 10^{-7} \mathrm{GeV}^{-2}}{\left\langle\sigma_{\mathrm{eff}} v\right\rangle\left(T_{3 / 2}\right)}\right)\left(\frac{m_{3 / 2}}{10^{4} \mathrm{TeV}}\right)^{-3 / 2}
\end{aligned}
$$

where we used Hubble parameter $H(T)=\sqrt{g_{*}(T) \pi^{2} / 90} T^{2} / M_{p}$, entropy density $s(T)=$ $2 \pi^{2} g_{*, s}(T) T^{3} / 45$. We also assumed $g_{*} \simeq g_{s, *}$ for the temperature of interest. We will present a more precise numerical evaluation in the following section.

For a lighter gravitino within the mass range, $10 \mathrm{TeV}<m_{3 / 2}<10^{4} \mathrm{TeV}$, the gravitino starts to decay at such a low temperature that the annihilation of wino DM is ineffective. In this case, almost all the winos produced from gravitino decays survive and hence, its relic abundance is proportional to the total gravitino abundance.

$$
\begin{aligned}
\Omega_{\tilde{W}}^{(\text {no-ann })} h^{2} & =\frac{m_{\tilde{W}}}{m_{3 / 2}}\left(\Omega_{3 / 2}^{U V} h^{2}+\Omega_{3 / 2}^{F I} h^{2}\right) \\
& \approx 0.12\left(\frac{m_{\tilde{W}}}{1 \mathrm{TeV}}\right)\left[\left(\frac{T_{R}}{2 \times 10^{9} \mathrm{GeV}}\right)+10^{-3}\left(\frac{100 \mathrm{TeV}}{m_{3 / 2}}\right)^{2} \sum_{i} g_{i}\left(\frac{m_{i}}{1000 \mathrm{TeV}}\right)^{3}\right],
\end{aligned}
$$

\footnotetext{
${ }^{3}$ We keep factors involving $\partial \log g_{*(s)}(T) / \partial \log T$ in the Boltzmann equation for $\rho_{\text {rad }}$.

${ }^{4}$ This version was kindly provided by Andrzej Hryczuk to JF in a previous project.
} 
where the first(second) term in the square brackets in the second line originates from decays of gravitino produced by the thermal scattering (freeze-in). We want to caution the reader that there is no sharp boundary value of $m_{3 / 2}$ that separates the two cases with "effective" and "ineffective" wino annihilations in eq. (2.8) and eq. (2.10). In section 3, we will derive more precise bounds by solving the Boltzmann equations numerically.

From eq. (2.10), we could see that for gravitino at or below PeV scale as in the mini-split scenario, to avoid overproduction of DM from gravitino decays, the reheating temperature has to be below

$$
T_{R} \lesssim 2 \times 10^{9} \mathrm{GeV}\left(\frac{1 \mathrm{TeV}}{m_{\tilde{W}}}\right)
$$

assuming a negligible contribution from freeze-in. This upper bound would only be pushed even lower if the freeze-in contribution is comparable to or even dominate over the thermal scattering contribution. Similarly, one could obtain an upper bound on the scalar soft mass

$$
m_{s} \lesssim 10^{4} \mathrm{TeV}\left(\frac{m_{3 / 2}}{100 \mathrm{TeV}}\right)^{2 / 3}\left(\frac{1 \mathrm{TeV}}{m_{\tilde{W}}}\right)^{1 / 3}
$$

Early discussions of reheating temperature in high-scale SUSY scenario with a decaying gravitino could be found in $[51,52]$.

\section{Indirect detection constraints}

As wino DM has a large annihilation rate, there are strong constraints on its relic abundance from indirect detection searches looking for its annihilation products [53-57]. Thus in the wino DM case, one could obtain a stronger upper bound on the reheating temperature compared to eq. (2.11) which holds for generic neutralino DM. In this section, we present a numerical evaluation of the constraints on the reheating temperature and SUSY scalar mass scale in the scenario with wino as (a component of) DM.

There are multiple indirect search channels for wino DM [58]. In general DM indirect detection searches for decay and annihilation products of DM in fluxes of cosmic rays containing charged particles or photons or neutrinos. We focus on searches looking for excesses in the photon continuum spectrum of satellite dwarf galaxies [53, 57], or our galactic center [59] and monochromatic photon line $[55,60] .{ }^{5}$ A continuum photon spectrum is generated from either the bremsstrahlung of charged particles or the hadronic fragmentation of the decay products of $W / Z$ 's in the final state of tree-level processes $\chi^{0} \chi^{0} \rightarrow W^{+} W^{-} / Z Z$. The gamma ray lines are generated from DM annihilation into $\gamma \gamma / \gamma Z$. Each photon in the final state carries away energy about the DM mass.

As demonstrated by figure 4 in ref. [23], the thermal wino relic abundance (computed in $[47,61]$ ) is ruled out by the indirect constraint for $m_{\tilde{W}}$ above $1.5 \mathrm{TeV}$ assuming standard cuspy (NFW and Einasto) DM halo profiles. Since the wino relic abundance is a sum of

\footnotetext{
${ }^{5}$ The first paper on the HESS search constraint for wino DM is ref. [61].
} 

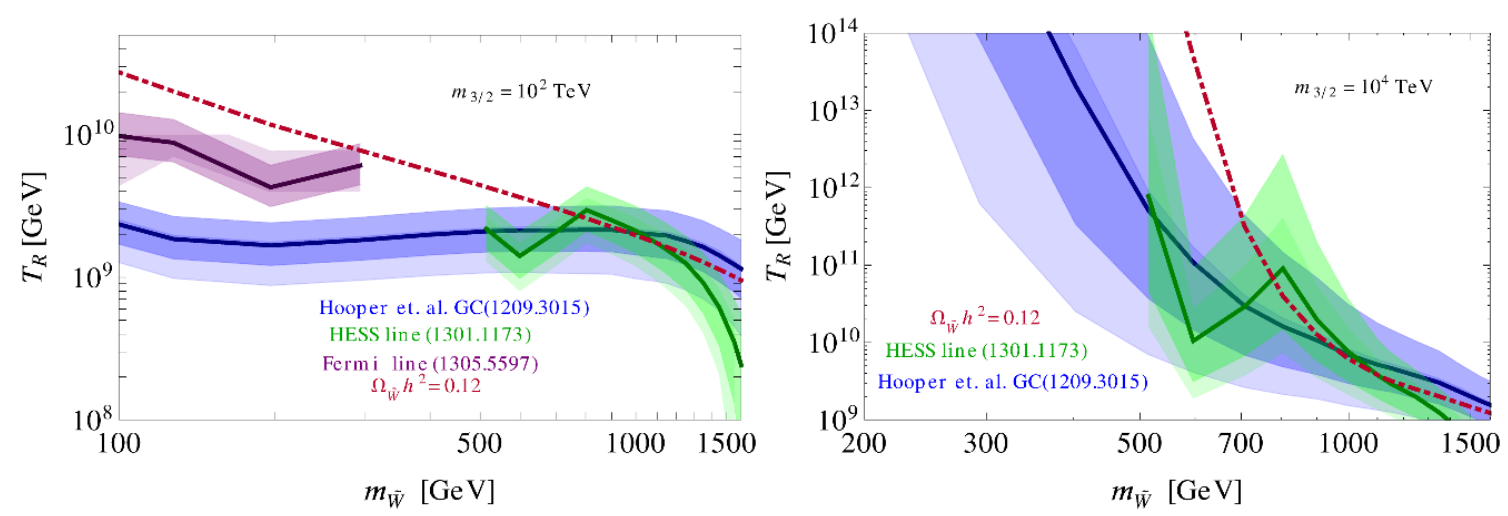

Figure 1. Upper bounds on inflaton reheating temperature $T_{R}$ as a function of wino mass for $m_{3 / 2}=100 \mathrm{TeV}$ (left) and $m_{3 / 2}=10^{4} \mathrm{TeV}$ (right). The blue, purple, green curves with bands around them correspond to constraints from Fermi galactic center continuum, Fermi line search and HESS line search respectively. The bands are derived by varying parameters of NFW (Einasto) dark matter profiles in the $2 \sigma$ range [65]. The burgundy dot-dashed line corresponds to the upper bound derived from requiring $\Omega_{\tilde{W}} h^{2}=0.12$.

the thermal contribution and the non-thermal contribution from gravitino decays, there is room for a non-thermal relic abundance only for wino with mass below $1.5 \mathrm{TeV} .{ }^{6}$

We express the constraints on allowed non-thermal $\Omega_{\tilde{W}} h^{2}$ as an upper bound on the inflaton reheating temperature $T_{R}$ as a function of wino mass for $m_{3 / 2}=100 \mathrm{TeV}$ and $10^{4} \mathrm{TeV}$ in figure 1 . In this figure, we assumed that freeze-in contribution to the primordial gravitino relic abundance is negligible. As mentioned at the end of last section, taking into account of the freeze-in contribution will only make the upper bound stronger.

The left panel of figure 1 stays almost unmodified for $10 \mathrm{TeV}<m_{3 / 2}<10^{4} \mathrm{TeV}$ as the wino annihilation is ineffective and the relic abundance is independent of $m_{3 / 2}$ as can be seen from the first term in eq. (2.10). The reheating temperature is bounded to be below $3 \times 10^{9} \mathrm{GeV}$ for the whole wino mass range. For wino mass close to $1.5 \mathrm{TeV}$, the HESS constraint pushes the reheating temperature to be even lower to about a few times $10^{8} \mathrm{GeV}$.

In the right panel of figure 1 , the gravitino mass is set to be $10^{4} \mathrm{TeV}$. In this case, for light wino with mass below $300 \mathrm{GeV}$, wino annihilation becomes effective and its relic abundance is insensitive to the reheating temperature as shown in eq. (2.8). Therefore, the upper bound on the inflaton reheating temperature is lifted up entirely. For heavier wino, the annihilation rate drops with the increasing mass and the wino relic abundance interpolates between eq. (2.8) and eq. (2.10). In the whole wino mass range, the upper bound on $T_{R}$ is above $10^{9} \mathrm{GeV}$. For even heavier gravitino, the bound on $T_{R}$ becomes even weaker.

One could also consider upper bound on the SUSY scalar masses, $m_{s}$, which is depicted in figure 2. In the left panel, we took $m_{3 / 2}=100 \mathrm{TeV}$ and $T_{R}=10^{8} \mathrm{GeV}$ so that the thermal

\footnotetext{
${ }^{6}$ There could be different non-thermal scenario such as moduli scenario [62]. The implications of indirect detection for moduli scenario have been discussed in [23, 63, 64].
} 

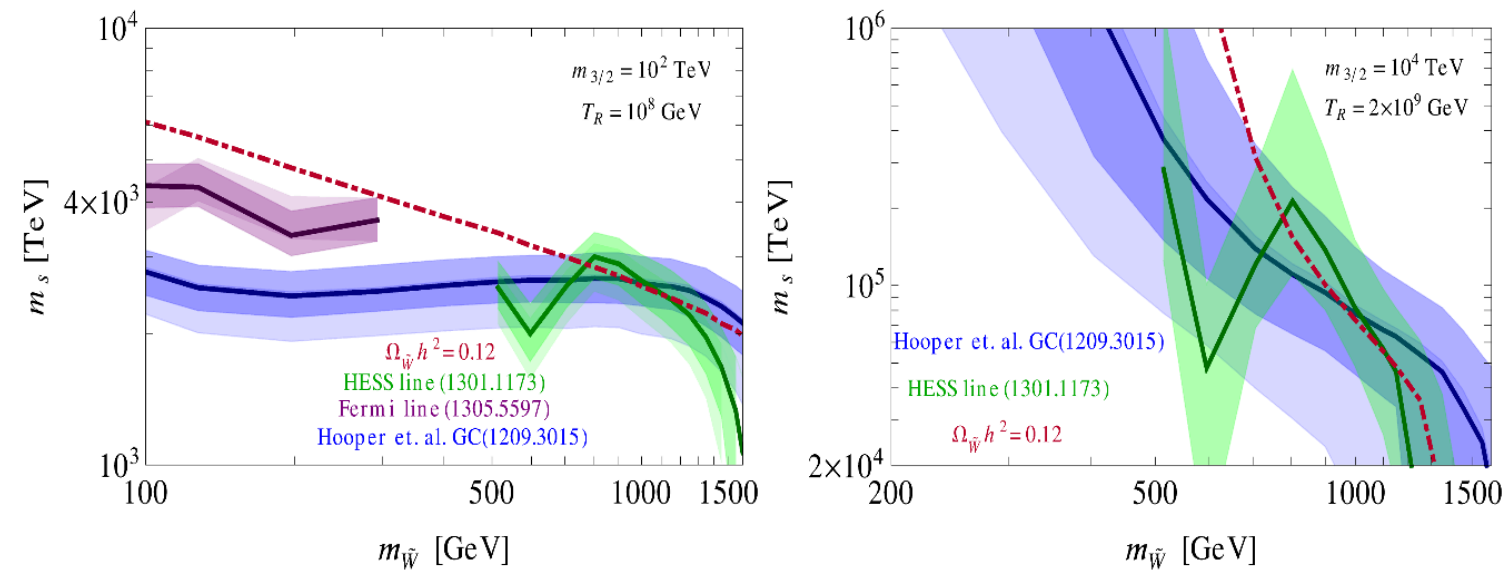

Figure 2. Upper bounds on scalar mass $m_{s}$ as a function of wino mass for $m_{3 / 2}=100 \mathrm{TeV}$ (left) and $m_{3 / 2}=10^{4} \mathrm{TeV}$ (right). The blue, purple, green curves with bands around them correspond to constraints from Fermi galactic center continuum, Fermi line search and HESS line search respectively. The bands are derived by varying parameters of NFW (Einasto) dark matter profiles in the $2 \sigma$ range [65]. The burgundy dot-dashed line corresponds to the upper bound derived from requiring $\Omega_{\tilde{W}} h^{2}=0.12$.

scattering contribution is negligible. Increasing the reheating temperature will only make the bound even stronger. In this case, indirect detection constraints restrict the scalar mass to be below $(2-3) \times 10^{3} \mathrm{TeV}$ for the whole wino mass range. In the right panel, we set $m_{3 / 2}=10^{4} \mathrm{TeV}$ and $T_{R}=2 \times 10^{9} \mathrm{GeV}$. Since this is the case where wino annihilation becomes more effective, the upper bounds on the SUSY scalar mass depends less on $T_{R}$ and are reduced significantly compared to the case with a lighter gravitino. More specifically, for wino above $300 \mathrm{GeV}$, indirect detection constraints restrict scalar masses to be below $10^{4}-10^{6} \mathrm{TeV}$. For wino below $300 \mathrm{GeV}$, the upper bound is almost lifted up entirely.

\section{Implications of the BICEP2 result}

Recently the BICEP2 collaboration reported a groundbreaking discovery of inflationary gravitational waves in the $B$-mode power spectrum in the range $30<l<150$ [24]. The observed $B$-mode spectrum is well fit by a lensed $\Lambda$-CDM plus tensor model with a tensorto-scalar ratio $r=0.20_{-0.05}^{+0.07}$. Such a large tensor-to-scalar ratio has a profound implication for the inflation paradigm. Notice that if running of the spectral index is allowed, the combined Planck and BICEP data could have a different best fit. In our paper, we will not explore this possibility as we don't expect $r$ to change much. We will first review the basics of tensor-to-scalar ratio in the slow-roll inflation paradigm for completeness in section 4.1. Readers who are familiar with this topic could skip this section. Then we will discuss the implications of BICEP2 result for the inflation mass scale and reheating temperature in section 4.2 . 


\subsection{Basics of tensor-to-scalar ratio}

We will follow closely Lecture 2 in ref. [66] in this brief review. In slow-roll inflation models, the metric perturbation during the inflation period could be decomposed into scalar and tensor modes, which result in density and gravitational wave fluctuations respectively. Each mode could be characterized by a fluctuation amplitude squared $[67,68]$

$$
\begin{aligned}
& \Delta_{s}^{2}(k)=\frac{H^{4}}{4 \pi^{2} \dot{\phi}^{2}}=\frac{1}{12 \pi^{2} M_{p}^{6}} \frac{V^{3}}{V^{\prime 2}} \quad \text { scalar } \\
& \Delta_{t}^{2}(k)=\frac{2 H^{2}}{\pi^{2} M_{p}^{2}}=\frac{2}{3 \pi^{2}} \frac{V}{M_{p}^{4}}, \quad \text { tensor }
\end{aligned}
$$

where the reduced Planck scale is $M_{p}=2.4 \times 10^{18} \mathrm{GeV}$. It should be understood that all the physical quantities above are evaluated at horizon crossing $k=a H$ at which the relevant comoving scales for the CMB exits the Hubble radius. $\dot{\phi}$ is the time derivative of the inflaton field $\phi$ and $V^{\prime}$ is the derivative of the inflaton potential with respect to $\phi$. In deriving the second expression of the amplitude squared in each line, we used equation of motion for the inflaton $H^{2} \approx V /\left(3 M_{p}^{2}\right)$ and $\dot{\phi} \approx-V^{\prime} /(3 H) .^{7}$

Normalizing the scalar spectrum to the COBE [70] or WMAP [71] anisotropy measurement gives $\Delta_{s}^{2}(k) \approx 2.2 \times 10^{-9}$. Then one could define tensor-to-scalar ratio $r \equiv$ $\Delta_{t}^{2}(k) / \Delta_{s}^{2}(k)$, which directly measures the inflation energy scale

$$
V \approx\left(1.8 \times 10^{16} \mathrm{GeV}\right)^{4}\left(\frac{r}{0.1}\right)
$$

$r$ also relates directly to the evolution of the inflaton as

$$
r=\frac{8}{M_{p}^{2}}\left(\frac{d \phi}{d N}\right)^{2},
$$

where differential $e$-folds $d N=H d t$. Then one could write the field displacement between the time when CMB fluctuations exited the horizon at $N_{\mathrm{cmb}}$ and the end of inflation at $N_{\text {end }}$ in terms of an integral

$$
\frac{\Delta \phi}{M_{p}}=\int_{N_{\mathrm{end}}}^{N_{\mathrm{cmb}}} d N \sqrt{\frac{r}{8}}
$$

Setting $N_{\mathrm{end}}=0$ and given that $N_{\mathrm{cmb}} \approx(40-60)$ and $r$ is approximately constant during the inflation era, one obtains the famous Lyth bound [72]

$$
\frac{\Delta \phi}{M_{p}} \approx 6.7\left(\frac{N_{\mathrm{cmb}}}{60}\right) \sqrt{\frac{r}{0.1}} .
$$

\footnotetext{
${ }^{7}$ One easy way to understand the appearance of $\dot{\phi}^{2}$ in the scalar perturbation amplitude squared is through effective field theory (EFT) [69]. The key insight of inflation EFT is that the inflaton spontaneously breaks time translation invariance and results in a Goldstone mode "eaten" by the graviton to appear in the scalar modes. Compared to the tensor mode, the kinetic term for the Goldstone (scalar) mode has an additional factor of $\dot{H}$ in the kinetic term, which signals the break down of EFT in the limit of pure de Sitter space $\dot{H}=0$. By equation of motion, $\dot{H}$ is proportional to $\dot{\phi}^{2}$ and consequently $\dot{\phi}^{2}$ appear in the denominator of scalar fluctuation amplitude squared.
} 
Inspecting eq. (4.3) and (4.6), one could see immediately that the BICEP2 result points towards a large field displacement of order Planck scale during inflation or in other words, large field inflation. Existing examples of large-field inflation include chaotic inflation where a single power term dominates the potential $[73,74]$

$$
V(\phi)=\lambda_{p} \phi^{p}
$$

and natural inflation with a periodic potential resulting from a shift symmetry the inflaton enjoys [75]

$$
V(\phi)=V_{0}\left(1+\cos \left(\frac{\phi}{f}\right)\right) .
$$

\subsection{Implication for reheating temperature}

Now we want to estimate the inflaton mass scale. We start with a toy model of large field inflation $V=m_{\phi}^{2} \phi^{2}$. In this model, the scalar fluctuation amplitude squared is

$$
\Delta_{s}^{2}(k)=\frac{m_{\phi}^{2}}{M_{p}^{2}} \frac{N_{\mathrm{cmb}}^{2}}{3 \pi^{2}}
$$

where $N_{\mathrm{cmb}}=\phi_{\mathrm{cmb}}^{2} /\left(4 M_{p}^{2}\right)$. Given the normalization to the CMB measurement, $\Delta_{s}^{2}(k) \approx$ $2.2 \times 10^{-9}$, the inflaton mass is

$$
m_{\phi} \approx 10^{13} \mathrm{GeV}\left(\frac{60}{N_{\mathrm{cmb}}}\right)^{2}
$$

One could check in more realistic models such as chaotic inflation and natural inflation that the inflaton mass scale is around $10^{13} \mathrm{GeV}[25,76,77]$. One crude estimate of the inflaton mass in all these large-field inflation model is

$$
m_{\phi}^{2} \sim \frac{V}{(\Delta \phi)^{2}} \approx\left(2 \times 10^{13} \mathrm{GeV}\right)^{2}
$$

where we used eq. (4.3) and (4.6) assuming $N_{\mathrm{cmb}}=60$.

After inflation ends, inflaton starts to oscillate around the minimal of the potential. Its coupling to other particles induce conversion of the inflationary energy into the SM degrees of freedom. The reheating temperature is then determined by the inflaton decay width $\Gamma_{\phi}$ as

$$
T_{R}=\left(\frac{10}{g_{*}\left(T_{R}\right) \pi^{2}}\right)^{1 / 4} \sqrt{\Gamma_{\phi} M_{p}} \approx 0.3 \sqrt{\Gamma_{\phi} M_{p}}
$$

where we took $g_{*}\left(T_{R}\right) \approx 200$. The simplest possibility is that inflatons decay through renormalizable couplings to lighter degrees of freedom. For example, the decay width is $\Gamma_{\phi}=y^{2} m_{\phi} /(8 \pi)$ for inflaton coupling to fermions with a Yukawa coupling $y$. Then the reheating temperature is

$$
T_{R} \approx 3 \times 10^{11} \mathrm{GeV}\left(\frac{y}{10^{-3}}\right) \sqrt{\frac{m_{\phi}}{10^{13} \mathrm{GeV}}} .
$$


Notice that Yukawa coupling larger than $10^{-5}$ only makes sense in supersymmetric scenarios where the one-loop quantum correction does not modify the inflaton potential much due to a cancelation between fermionic and bosonic contributions.

If the renormalizable couplings of inflaton to lighter particles are negligible (e.g., $y<$ $10^{-5}$ ), it would always decay through Planck-scale suppressed operators. At the leading order, the inflaton decay width and the corresponding reheating temperature are

$$
\Gamma_{\phi}=\frac{c m_{\phi}^{3}}{M_{p}^{2}}, \quad T_{R} \approx 5 \times 10^{9} \mathrm{GeV} \sqrt{c}\left(\frac{m_{\phi}}{10^{13} \mathrm{GeV}}\right)^{3 / 2},
$$

where $c$ is some order one number determined by quantum gravity. From the point of view of operator analysis, this decay is induced by dimension five operators such as $\phi F \tilde{F} / M_{p}$ with $F$ the field strength of SM gauge interaction. In other words, the BICEP2 results imply a minimal reheating temperature at or above $10^{9} \mathrm{GeV}$ !

One should worry about the caveats of the very simple estimate above. One question is whether the leading order gravitational couplings through dimension five operators could be suppressed and the reheating temperature could be even lower. This could be true if the inflaton is charged under a gauge symmetry (global symmetry is not respected by quantum gravity) and then dimension five operators are forbidden. This is an interesting possibility but we will not explore it here further but leave it for future work. Another concern is that since reheating is a very complicated process (for a review, see [78]), our simple estimate of a minimal reheating temperature might be misleading. In particular, there could exist a preheating era in which particles coupled to the inflaton are resonantly produced by parametric resonance and the temperature of the plasma could be higher than the reheating temperature. Yet preheating might make the tension between the upper bound on $T_{R}$ derived in section 2 and section 3 and the lower bound on $T_{R}$ derived in this section even worse. The reason is that gravitinos could be over-produced non-thermally during the preheating era [79-83]. ${ }^{8}$ Nonetheless, it is interesting and important to carry out a thorough study of preheating/reheating in sound (stringy) inflation models.

\section{Implications for SUSY}

So far we have demonstrated a (mild) tension between mini-split SUSY with a heavy unstable gravitino at around the PeV scale and the BICEP2 result. In mini-split SUSY, the reheating temperature has to be below $10^{9} \mathrm{GeV}$ to avoid overproduction of DM particles from gravitino decay while the BICEP2 result prefers large-field inflation with a reheating temperature above $10^{9} \mathrm{GeV}$. In other words, the BICEP2 results favor a larger splitting between the gravitino and the gauginos than the one-loop factor if the gauginos are fixed at around the $\mathrm{TeV}$ scale. Interestingly, the requirement that gaugino mass does not exceed the TeV scale constrains the gravitino mass to be around or below $10^{13} \mathrm{GeV}$, which is also the mass scale of the inflaton implied by BICEP2! Below we will review the derivation of this statement by operator analysis following refs. [10, 11].

\footnotetext{
${ }^{8}$ In certain supergravity models, the non-thermal production could be suppressed [84, 85].
} 
In supergravity, the easiest way to cancel the positive vacuum energy from SUSY breaking contribution is to have a non-zero VEV of the superpotential. As the superpotential $W$ carries $R$-charge 2 , its VEV $W_{0}$ breaks $\mathrm{U}(1)_{\mathrm{R}}$ symmetry spontaneously. It also gives a gravitino mass

$$
m_{3 / 2} \approx \frac{W_{0}}{M_{p}^{2}} \approx \frac{\left|F_{X}\right|}{\sqrt{3} M_{p}},
$$

where $F_{X}$ is the $F$-term VEV of the SUSY breaking spurion $X$. A non-zero gaugino mass is generated only when both $\mathrm{U}(1)_{\mathrm{R}}$ and SUSY are broken. The lowest dimensional operator built out of SUSY breaking spurion $X, \mathrm{U}(1)_{\mathrm{R}}$ breaking spurion $W$ and MSSM superfields arise in the Kähler potential

$$
\int d^{2} \theta d^{2} \bar{\theta} \frac{X^{\dagger} X W W_{\alpha} W^{\alpha}}{M_{*}^{6}}
$$

where $W_{\alpha}$ denotes the MSSM gauge supermultiplet. This operator could be generated by gravitational loops where $M_{*} \sim M_{p}$ and gives a minimal contribution to the gaugino mass

$$
m_{1 / 2} \gtrsim \frac{\left|F_{X}\right|^{2} W_{0}}{M_{p}^{6}} \approx \frac{3 m_{3 / 2}^{3}}{M_{p}^{2}} .
$$

Requiring $m_{1 / 2}$ at or below TeV leads to $m_{3 / 2} \lesssim 10^{13} \mathrm{GeV}$ ! This large hierarchy between gravitino and gaugino could be realized in no-scale supergravity which could arise from the Scherk-Schwarz mechanism [86, 87].

\section{Conclusions and outlook}

In this paper, we study the implication of DM indirect detection and BICEP2 in the split SUSY scenario with a heavy unstable gravitino. In the mini-split spectrum with scalars/gravitinos only one-loop factor above the $\mathrm{TeV}$-scale gauginos, the reheating temperature has to be low to avoid overproduction of DM particles from gravitino decays. In particular, we demonstrate that indirect detection requires the reheating temperature to be below about $10^{9} \mathrm{GeV}$ if the wino is (a component of) DM. On the other hand, the large tensor-to-scalar ratio observed by BICEP2 favors large-field-inflation with a reheating temperature around or above $10^{9} \mathrm{GeV}$. Given this mild tension and the phenomenological upper bound on the gravitino mass derived by requiring the gauginos to be at the $\mathrm{TeV}$ scale, it is tempting to think more seriously of the (highly) split SUSY scenario in which inflaton/gravitino are at around $10^{13} \mathrm{TeV}$ and gauginos are still at the $\mathrm{TeV}$ scale with lightest neutralino being (part of) DM..$^{9}$ Indeed this picture has recently been discussed in the framework of Intermediate Scale SUSY [33].

In general, given the BICEP2 result, it is very interesting to use the scale of inflation to probe the full range of split SUSY scenarios through observables such as equilateral nongaussianity [29]. It will also be of interest to study the implications of the BICEP2 result for baryogenesis. For example, thermal leptogenesis works for a reheating temperature above $2 \times 10^{9} \mathrm{GeV}$ [88], which fits well with the BICEP2 result.

\footnotetext{
${ }^{9}$ Axion could be the dominant DM component.
} 


\section{Acknowledgments}

We thank Haipeng An, Joseph Bramante, Tim Cohen, Raffaele Tito D'Agnolo, Matt Reece, Kuver Sinha and Scott Watson for useful comments. JF thanks the Center for Future High Energy Physics in Beijing for hospitality where part of the project is carried out. JF does not thank the thief who stole her laptop before she could save the draft to dropbox in the final stage of the project.

\section{A Gravitino from inflaton decay}

In this appendix, we review non-thermal gravitino production from inflaton decays. In general, decays of inflaton can overproduce gravitinos which subsequent decays can induce LSP overproduction $[40,41]$. Consider the following simple model of SUSY breaking and inflation [43],

$$
\begin{aligned}
& K=|\phi|^{2}+|X|^{2}+|z|^{2}-\frac{|z|^{4}}{\tilde{\Lambda}^{2}}, \\
& W=X\left(g \frac{\phi^{n}}{M_{p}^{n-2}}-v^{2}\right)+\mu^{2} z+W_{0},
\end{aligned}
$$

where $z$ is the SUSY breaking spurion and $\tilde{\Lambda}$ is the QCD scale of the dynamical SUSY breaking sector. Here, $\mu$ is the SUSY breaking scale related to the $F$-term VEV of $z$ through $F_{z} \simeq-\mu^{2} \simeq \sqrt{3} m_{3 / 2} M_{p}$ and $W_{0}$ is the constant term introduced in order to cancel the positive vacuum energy from SUSY breaking in order to obtain a vanishing cosmological constant.

The scalar potential in supergravity is given by

$$
V=e^{K / M_{p l}^{2}}\left[K_{i \bar{j}}^{-1}\left(D_{i} W\right)\left(D_{\bar{j}} W\right)-3 \frac{|W|^{2}}{M_{p}^{2}}\right],
$$

where $D_{i}$ is the covariant derivative with respect to field $i$. There is a mass mixing between $X$ and $z$ arising from the following terms in the scalar potential above (A.3),

$$
V \supset\left|\frac{X n g \phi^{n-1}}{M_{p}^{n-2}}+\frac{\phi^{\dagger} W}{M_{p}^{2}}\right|^{2} \approx \frac{m_{\phi}\langle\phi\rangle \mu^{2}}{M_{p}^{2}} X z^{\dagger}+h . c,
$$

where $m_{\phi}=n g\langle\phi\rangle^{n-1} / M_{p}^{n-2}$.

The operator $|z|^{4} / \tilde{\Lambda}$ in the Kähler potential induces $z$ decaying into the goldstino pair $(\tilde{z})$ via

$$
\mathcal{L} \supset \int d^{2} \theta d^{2} \bar{\theta} K \sim-2 \frac{F_{z}^{\dagger}}{\tilde{\Lambda}^{2}} z^{\dagger} \tilde{z} \tilde{z}+h . c
$$

where the decay rate is given by

$$
\Gamma_{z \rightarrow \tilde{z} \tilde{z}} \simeq \frac{1}{96 \pi} \frac{m_{z}^{5}}{m_{3 / 2}^{2} M_{p}^{2}} .
$$


Since the goldstino is "eaten" by the gravitino via the super-Higgs mechanism, the decay rate above can be expressed as the decay rate of the inflaton into a pair of gravitinos via the mass mixing with $z$ :

$$
\begin{aligned}
\Gamma_{\phi \rightarrow \tilde{z} \tilde{z}} & \sim\left(\frac{\theta}{\sqrt{2}}\right)^{2} \frac{m_{\phi}}{m_{z}} \Gamma_{z \rightarrow \tilde{z} \tilde{z}}, \\
& \sim\left(\frac{\theta}{\sqrt{2}}\right)^{2}\left(\frac{m_{z}}{m_{\phi}}\right)^{4} \frac{m_{\phi}^{5}}{m_{3 / 2}^{2} M_{p}^{2}}
\end{aligned}
$$

where the mixing angle between inflaton $\phi$ and $z, \theta$, is given by $\sqrt{3}\left(m_{3 / 2}\langle\phi\rangle\right) /\left(m_{\phi} M_{p}\right)$ for $m_{\phi} \gg m_{z}, \sqrt{3}\left(m_{3 / 2} m_{\phi}\langle\phi\rangle\right) /\left(m_{z}^{2} M_{p}\right)$ for $m_{\phi} \ll m_{z}$. Therefore, in the case that $m_{\phi} \gg m_{z}$, the decay rate (A.7) of inflaton into a pair of gravitino is suppressed by $\left(m_{z} / m_{\phi}\right)^{4}$.

If $z$ is only charged under some global symmetry, one could not forbid operators such as $|\phi|^{2} z$ and $|\phi|^{2} z z$ in the Kähler potential (A.1). These operators will always be induced by Planck scale physics as it only respects local symmetries [89]. These operators are dangerous as they would enhance the decay rate of inflaton to gravitinos by $m_{\phi}^{2} / m_{3 / 2}^{2}$. Thus in addition to the hierarchy $m_{3 / 2} \ll m_{z} \ll m_{\phi}$, the SUSY breaking spurion cannot be a gauge singlet!

Open Access. This article is distributed under the terms of the Creative Commons Attribution License (CC-BY 4.0), which permits any use, distribution and reproduction in any medium, provided the original author(s) and source are credited.

\section{References}

[1] G.F. Giudice, M.A. Luty, H. Murayama and R. Rattazzi, Gaugino mass without singlets, JHEP 12 (1998) 027 [hep-ph/9810442] [INSPIRE].

[2] L. Randall and R. Sundrum, Out of this world supersymmetry breaking, Nucl. Phys. B 557 (1999) 79 [hep-th/9810155] [INSPIRE].

[3] K. Choi, A. Falkowski, H.P. Nilles and M. Olechowski, Soft supersymmetry breaking in KKLT flux compactification, Nucl. Phys. B 718 (2005) 113 [hep-th/0503216] [INSPIRE].

[4] K. Choi, K.S. Jeong and K.-i. Okumura, Phenomenology of mixed modulus-anomaly mediation in fluxed string compactifications and brane models, JHEP 09 (2005) 039 [hep-ph/0504037] [INSPIRE].

[5] J.P. Conlon and F. Quevedo, Gaugino and Scalar Masses in the Landscape, JHEP 06 (2006) 029 [hep-th/0605141] [INSPIRE].

[6] J.P. Conlon, S.S. AbdusSalam, F. Quevedo and K. Suruliz, Soft SUSY Breaking Terms for Chiral Matter in IIB String Compactifications, JHEP 01 (2007) 032 [hep-th/0610129] [INSPIRE].

[7] B.S. Acharya, K. Bobkov, G.L. Kane, P. Kumar and J. Shao, Explaining the Electroweak Scale and Stabilizing Moduli in M-theory, Phys. Rev. D 76 (2007) 126010 [hep-th/0701034] [INSPIRE].

[8] B.S. Acharya, K. Bobkov, G.L. Kane, J. Shao and P. Kumar, The $G_{2}$-MSSM: An M-theory motivated model of Particle Physics, Phys. Rev. D 78 (2008) 065038 [arXiv:0801.0478] [INSPIRE]. 
[9] J.D. Wells, Implications of supersymmetry breaking with a little hierarchy between gauginos and scalars, hep-ph/0306127 [INSPIRE].

[10] N. Arkani-Hamed and S. Dimopoulos, Supersymmetric unification without low energy supersymmetry and signatures for fine-tuning at the LHC, JHEP 06 (2005) 073 [hep-th/0405159] [INSPIRE].

[11] N. Arkani-Hamed, S. Dimopoulos, G.F. Giudice and A. Romanino, Aspects of split supersymmetry, Nucl. Phys. B 709 (2005) 3 [hep-ph/0409232] [INSPIRE].

[12] G.F. Giudice and A. Romanino, Split supersymmetry, Nucl. Phys. B 699 (2004) 65 [Erratum ibid. B 706 (2005) 65] [hep-ph/0406088] [INSPIRE].

[13] L.J. Hall and Y. Nomura, Spread Supersymmetry, JHEP 01 (2012) 082 [arXiv:1111.4519] [INSPIRE].

[14] M. Ibe and T.T. Yanagida, The Lightest Higgs Boson Mass in Pure Gravity Mediation Model, Phys. Lett. B 709 (2012) 374 [arXiv:1112.2462] [INSPIRE].

[15] A. Arvanitaki, N. Craig, S. Dimopoulos and G. Villadoro, Mini-Split, JHEP 02 (2013) 126 [arXiv: 1210.0555] [INSPIRE].

[16] N. Arkani-Hamed, A. Gupta, D.E. Kaplan, N. Weiner and T. Zorawski, Simply Unnatural Supersymmetry, arXiv:1212.6971 [INSPIRE].

[17] L.J. Hall, Y. Nomura and S. Shirai, Spread Supersymmetry with Wino LSP: Gluino and Dark Matter Signals, JHEP 01 (2013) 036 [arXiv:1210.2395] [INSPIRE].

[18] L.J. Hall and Y. Nomura, Grand Unification and Intermediate Scale Supersymmetry, JHEP 02 (2014) 129 [arXiv:1312.6695] [INSPIRE].

[19] W. Altmannshofer, R. Harnik and J. Zupan, Low Energy Probes of PeV Scale Sfermions, JHEP 11 (2013) 202 [arXiv:1308.3653] [InSPIRE].

[20] M. Baumgart, D. Stolarski and T. Zorawski, Split SUSY Radiates Flavor, arXiv:1403.6118 [INSPIRE].

[21] M. Dhuria and A. Misra, (N)LSP Decays and Gravitino Dark Matter Relic Abundance in Big Divisor (nearly) SLagy D3/D7 mu-Split SUSY, Nucl. Phys. B 867 (2013) 636 [arXiv:1207.2774] [INSPIRE].

[22] T. Cohen, M. Lisanti, A. Pierce and T.R. Slatyer, Wino Dark Matter Under Siege, JCAP 10 (2013) 061 [arXiv:1307.4082] [INSPIRE].

[23] J. Fan and M. Reece, In Wino Veritas? Indirect Searches Shed Light on Neutralino Dark Matter, JHEP 10 (2013) 124 [arXiv:1307.4400] [INSPIRE].

[24] BICEP2 collaboration, P.A.R. Ade et al., Detection of B-Mode Polarization at Degree Angular Scales by BICEP2, Phys. Rev. Lett. 112 (2014) 241101 [arXiv:1403.3985] [INSPIRE].

[25] K. Harigaya and T.T. Yanagida, Discovery of Large Scale Tensor Mode and Chaotic Inflation in Supergravity, arXiv:1403.4729 [INSPIRE].

[26] C. Pallis, Induced-Gravity Inflation in no-Scale Supergravity and Beyond, arXiv:1403.5486 [INSPIRE].

[27] K. Harigaya, M. Ibe, K. Ichikawa, K. Kaneta and S. Matsumoto, High-Scale SUSY Breaking Models in light of the BICEP2 Result, arXiv:1403.5880 [INSPIRE]. 
[28] L.E. Ibáñez and I. Valenzuela, BICEP2, the Higgs Mass and the SUSY-breaking Scale, arXiv:1403.6081 [INSPIRE].

[29] N. Craig and D. Green, Testing Split Supersymmetry with Inflation, arXiv:1403.7193 [INSPIRE].

[30] J. Ellis, M.A.G. García, D.V. Nanopoulos and K.A. Olive, Resurrecting Quadratic Inflation in No-Scale Supergravity in Light of BICEP2, JCAP 05 (2014) 037 [arXiv:1403.7518] [INSPIRE].

[31] D.H. Lyth, BICEP2, the curvature perturbation and supersymmetry, arXiv:1403.7323 [INSPIRE].

[32] K. Hamaguchi, T. Moroi and T. Terada, Complexified Starobinsky Inflation in Supergravity in the Light of Recent BICEP2 Result, Phys. Lett. B 733 (2014) 305 [arXiv:1403.7521] [INSPIRE].

[33] L.J. Hall, Y. Nomura and S. Shirai, Grand Unification, Axion and Inflation in Intermediate Scale Supersymmetry, JHEP 06 (2014) 137 [arXiv:1403.8138] [INSPIRE].

[34] J. Pradler and F.D. Steffen, Constraints on the Reheating Temperature in Gravitino Dark Matter Scenarios, Phys. Lett. B 648 (2007) 224 [hep-ph/0612291] [INSPIRE].

[35] V.S. Rychkov and A. Strumia, Thermal production of gravitinos, Phys. Rev. D 75 (2007) 075011 [hep-ph/0701104] [INSPIRE].

[36] R. Allahverdi, A. Jokinen and A. Mazumdar, Gravitino production from reheating in split supersymmetry, Phys. Rev. D 71 (2005) 043505 [hep-ph/0410169] [INSPIRE].

[37] C. Cheung, G. Elor and L. Hall, Gravitino Freeze-In, Phys. Rev. D 84 (2011) 115021 [arXiv: 1103.4394] [INSPIRE].

[38] L.J. Hall, K. Jedamzik, J. March-Russell and S.M. West, Freeze-In Production of FIMP Dark Matter, JHEP 03 (2010) 080 [arXiv:0911.1120] [INSPIRE].

[39] C. Cheung, G. Elor, L.J. Hall and P. Kumar, Origins of Hidden Sector Dark Matter I: Cosmology, JHEP 03 (2011) 042 [arXiv: 1010.0022] [INSPIRE].

[40] M. Kawasaki, F. Takahashi and T.T. Yanagida, Gravitino overproduction in inflaton decay, Phys. Lett. B 638 (2006) 8 [hep-ph/0603265] [INSPIRE].

[41] M. Kawasaki, F. Takahashi and T.T. Yanagida, The Gravitino-overproduction problem in inflationary universe, Phys. Rev. D 74 (2006) 043519 [hep-ph/0605297] [INSPIRE].

[42] M. Dine, R. Kitano, A. Morisse and Y. Shirman, Moduli decays and gravitinos, Phys. Rev. D 73 (2006) 123518 [hep-ph/0604140] [INSPIRE].

[43] K. Nakayama, F. Takahashi and T.T. Yanagida, Eluding the Gravitino Overproduction in Inflaton Decay, Phys. Lett. B 718 (2012) 526 [arXiv:1209.2583] [INSPIRE].

[44] M. Kawasaki, K. Kohri, T. Moroi and A. Yotsuyanagi, Big-Bang Nucleosynthesis and Gravitino, Phys. Rev. D 78 (2008) 065011 [arXiv:0804.3745] [INSPIRE].

[45] T. Moroi, M. Nagai and M. Takimoto, Non-Thermal Production of Wino Dark Matter via the Decay of Long-Lived Particles, JHEP 07 (2013) 066 [arXiv: 1303. 0948] [INSPIRE].

[46] P. Gondolo, J. Edsjo, P. Ullio, L. Bergstrom, M. Schelke et al., DarkSUSY: Computing supersymmetric dark matter properties numerically, JCAP 07 (2004) 008 [astro-ph/0406204] [INSPIRE]. 
[47] J. Hisano, S. Matsumoto, M. Nagai, O. Saito and M. Senami, Non-perturbative effect on thermal relic abundance of dark matter, Phys. Lett. B 646 (2007) 34 [hep-ph/0610249] [INSPIRE].

[48] A. Hryczuk and R. Iengo, The one-loop and Sommerfeld electroweak corrections to the Wino dark matter annihilation, JHEP 01 (2012) 163 [Erratum ibid. 1206 (2012) 137] [arXiv:1111.2916] [INSPIRE].

[49] A. Hryczuk, The Sommerfeld enhancement for scalar particles and application to sfermion co-annihilation regions, Phys. Lett. B 699 (2011) 271 [arXiv:1102.4295] [INSPIRE].

[50] M. Ibe, S. Matsumoto and R. Sato, Mass Splitting between Charged and Neutral Winos at Two-Loop Level, Phys. Lett. B 721 (2013) 252 [arXiv:1212.5989] [INSPIRE].

[51] R. Kallosh, A. Linde, K.A. Olive and T. Rube, Chaotic inflation and supersymmetry breaking, Phys. Rev. D 84 (2011) 083519 [arXiv:1106.6025] [INSPIRE].

[52] E. Dudas, A. Linde, Y. Mambrini, A. Mustafayev and K.A. Olive, Strong moduli stabilization and phenomenology, Eur. Phys. J. C 73 (2013) 2268 [arXiv:1209.0499] [INSPIRE].

[53] Fermi-LAT collaboration, M. Ackermann et al., Constraining Dark Matter Models from a Combined Analysis of Milky Way Satellites with the Fermi Large Area Telescope, Phys. Rev. Lett. 107 (2011) 241302 [arXiv:1108.3546] [inSPIRE].

[54] Fermi-LAT collaboration, M. Ackermann et al., The Fermi Large Area Telescope On Orbit: Event Classification, Instrument Response Functions and Calibration, Astrophys. J. Suppl. 203 (2012) 4 [arXiv:1206.1896] [INSPIRE].

[55] Fermi-LAT collaboration, M. Ackermann et al., Search for Gamma-ray Spectral Lines with the Fermi Large Area Telescope and Dark Matter Implications, Phys. Rev. D 88 (2013) 082002 [arXiv: 1305.5597] [INSPIRE].

[56] H.E.S.S. collaboration, A. Abramowski et al., Search for a Dark Matter annihilation signal from the Galactic Center halo with H.E.S.S, Phys. Rev. Lett. 106 (2011) 161301 [arXiv: 1103.3266] [INSPIRE].

[57] A. Drlica-Wagner, Searching for dark matterin dwarf spheroidal satellite galaxies with the fermi-lat, http://fermi.gsfc.nasa.gov/science/mtgs/symposia/2012/program/fri/ADrlica-Wagner.pdf, 2012.

[58] A. Hryczuk, I. Cholis, R. Iengo, M. Tavakoli and P. Ullio, Indirect Detection Analysis: Wino Dark Matter Case Study, arXiv:1401.6212 [INSPIRE].

[59] D. Hooper, C. Kelso and F.S. Queiroz, Stringent and Robust Constraints on the Dark Matter Annihilation Cross Section From the Region of the Galactic Center, Astropart. Phys. 46 (2013) 55 [arXiv: 1209.3015] [INSPIRE].

[60] H.E.S.S. collaboration, A. Abramowski et al., Search for photon line-like signatures from Dark Matter annihilations with H.E.S.S, Phys. Rev. Lett. 110 (2013) 041301 [arXiv: 1301.1173] [INSPIRE].

[61] M. Cirelli, A. Strumia and M. Tamburini, Cosmology and Astrophysics of Minimal Dark Matter, Nucl. Phys. B 787 (2007) 152 [arXiv:0706.4071] [InSPIRE].

[62] T. Moroi and L. Randall, Wino cold dark matter from anomaly mediated SUSY breaking, Nucl. Phys. B 570 (2000) 455 [hep-ph/9906527] [INSPIRE]. 
[63] R. Easther, R. Galvez, O. Ozsoy and S. Watson, Supersymmetry, Nonthermal Dark Matter and Precision Cosmology, Phys. Rev. D 89 (2014) 023522 [arXiv:1307.2453] [INSPIRE].

[64] R. Allahverdi, M. Cicoli, B. Dutta and K. Sinha, Nonthermal dark matter in string compactifications, Phys. Rev. D 88 (2013) 095015 [arXiv:1307.5086] [INSPIRE].

[65] F. Iocco, M. Pato, G. Bertone and P. Jetzer, Dark Matter distribution in the Milky Way: microlensing and dynamical constraints, JCAP 11 (2011) 029 [arXiv:1107.5810] [INSPIRE].

[66] D. Baumann, TASI Lectures on Inflation, arXiv:0907.5424 [INSPIRE].

[67] V.F. Mukhanov, Gravitational Instability of the Universe Filled with a Scalar Field, JETP Lett. 41 (1985) 493 [INSPIRE].

[68] V.F. Mukhanov, Quantum Theory of Gauge Invariant Cosmological Perturbations, Sov. Phys. JETP 67 (1988) 1297 [INSPIRE].

[69] C. Cheung, P. Creminelli, A.L. Fitzpatrick, J. Kaplan and L. Senatore, The Effective Field Theory of Inflation, JHEP 03 (2008) 014 [arXiv:0709.0293] [INSPIRE].

[70] G.F. Smoot, C.L. Bennett, A. Kogut, E.L. Wright, J. Aymon et al., Structure in the COBE differential microwave radiometer first year maps, Astrophys. J. 396 (1992) L1 [INSPIRE].

[71] WMAP collaboration, D.N. Spergel et al., Wilkinson Microwave Anisotropy Probe (WMAP) three year results: implications for cosmology, Astrophys. J. Suppl. 170 (2007) 377 [astro-ph/0603449] [INSPIRE].

[72] D.H. Lyth, What would we learn by detecting a gravitational wave signal in the cosmic microwave background anisotropy?, Phys. Rev. Lett. 78 (1997) 1861 [hep-ph/9606387] [INSPIRE].

[73] A.D. Linde, eternal chaotic inflation, Mod. Phys. Lett. A 1 (1986) 81 [INSPIRE].

[74] A.D. Linde, Eternally Existing Selfreproducing Chaotic Inflationary Universe, Phys. Lett. B 175 (1986) 395 [INSPIRE].

[75] K. Freese, J.A. Frieman and A.V. Olinto, Natural inflation with pseudo - Nambu-Goldstone bosons, Phys. Rev. Lett. 65 (1990) 3233 [INSPIRE].

[76] K. Harigaya, M. Ibe, K. Schmitz and T.T. Yanagida, Dynamical Chaotic Inflation in the Light of BICEP2, Phys. Lett. B 733 (2014) 283 [arXiv:1403.4536] [INSPIRE].

[77] K. Freese and W.H. Kinney, Natural Inflation: Consistency with Cosmic Microwave Background Observations of Planck and BICEP2, arXiv:1403.5277 [INSPIRE].

[78] B.A. Bassett, S. Tsujikawa and D. Wands, Inflation dynamics and reheating, Rev. Mod. Phys. 78 (2006) 537 [astro-ph/0507632] [INSPIRE].

[79] R. Kallosh, L. Kofman, A.D. Linde and A. Van Proeyen, Gravitino production after inflation, Phys. Rev. D 61 (2000) 103503 [hep-th/9907124] [INSPIRE].

[80] G.F. Giudice, A. Riotto and I. Tkachev, Thermal and nonthermal production of gravitinos in the early universe, JHEP 11 (1999) 036 [hep-ph/9911302] [INSPIRE].

[81] R. Kallosh, L. Kofman, A.D. Linde and A. Van Proeyen, Superconformal symmetry, supergravity and cosmology, Class. Quant. Grav. 17 (2000) 4269 [Erratum ibid. 21 (2004) 5017] [hep-th/0006179] [INSPIRE].

[82] A.L. Maroto and A. Mazumdar, Production of spin $3 / 2$ particles from vacuum fluctuations, Phys. Rev. Lett. 84 (2000) 1655 [hep-ph/9904206] [INSPIRE]. 
[83] H.P. Nilles, M. Peloso and L. Sorbo, Coupled fields in external background with application to nonthermal production of gravitinos, JHEP 04 (2001) 004 [hep-th/0103202] [INSPIRE].

[84] H.P. Nilles, M. Peloso and L. Sorbo, Nonthermal production of gravitinos and inflatinos, Phys. Rev. Lett. 87 (2001) 051302 [hep-ph/0102264] [INSPIRE].

[85] P.B. Greene, K. Kadota and H. Murayama, Supergravity inflation free from harmful relics, Phys. Rev. D 68 (2003) 043502 [hep-ph/0208276] [InSPIRE].

[86] J.R. Ellis, C. Kounnas and D.V. Nanopoulos, No Scale Supersymmetric Guts, Nucl. Phys. B 247 (1984) 373 [INSPIRE].

[87] J. Scherk and J.H. Schwarz, How to Get Masses from Extra Dimensions, Nucl. Phys. B 153 (1979) 61 [INSPIRE].

[88] G.F. Giudice, A. Notari, M. Raidal, A. Riotto and A. Strumia, Towards a complete theory of thermal leptogenesis in the SM and MSSM, Nucl. Phys. B 685 (2004) 89 [hep-ph/0310123] [INSPIRE].

[89] M. Kamionkowski and J. March-Russell, Planck scale physics and the Peccei-Quinn mechanism, Phys. Lett. B 282 (1992) 137 [hep-th/9202003] [INSPIRE]. 